\title{
Quantum differential ghost microscopy
}

\author{
E. Losero $\odot,{ }^{1,2}$ I. Ruo-Berchera, ${ }^{1}$ A. Meda,${ }^{1}$ A. Avella, ${ }^{1}$ O. Sambataro,${ }^{3}$ and M. Genovese ${ }^{1,4}$ \\ ${ }^{1}$ Istituto Nazionale di Ricerca Metrologica (INRIM), Strada delle Cacce 91, 10135 Torino, Italy \\ ${ }^{2}$ Politecnico di Torino, Corso Duca degli Abruzzi 24, 10129 Torino, Italy \\ ${ }^{3}$ Dipartimento di Fisica, Università degli Studi di Torino, Via P. Giuria 1, 10125 Torino, Italy \\ ${ }^{4}$ INFN, sezione di Torino, via P. Giuria 1, 10125 Torino, Italy
}

(Received 18 June 2019; published 10 December 2019)

\begin{abstract}
Quantum correlations become formidable tools for beating classical capacities of measurement. Preserving these advantages in practical systems, where experimental imperfections are unavoidable, is a challenge of the utmost importance. Here we propose and realize a quantum ghost imaging protocol stemming from the differential ghost imaging, a scheme elaborated so far in the limit of bright thermal light, particularly suitable in the relevant case of faint or sparse objects. The extension toward the quantum regime represents an important step as quantum correlations allow low-brightness imaging, desirable for reducing the absorption dose. Furthermore, we optimize the protocol in terms of signal-to-noise ratio, to compensate for the detrimental effects of detection noise and losses. We perform the experiment using spontaneous parametric down conversion light in a microscope configuration. The image is reconstructed exploiting nonclassical intensity correlation in the low photon flux regime, rather than photon pairs detection coincidences. On the one side, we validate the theoretical model and on the other we show the applicability of this technique by imaging biological samples.
\end{abstract}

DOI: 10.1103/PhysRevA.100.063818

\section{INTRODUCTION}

Ghost imaging (GI) was theoretically proposed in 1994 [1] and experimentally demonstrated by Pittman et al. one year later [2] by using quantum correlations generated by spontaneous parametric down conversion (SPDC). It was considered as the earliest quantum imaging technique [3], but soon it has been shown that also classical correlations, as present in split thermal beams, can be successfully exploited, although with smaller visibility [4-10]. Two spatially correlated beams are used: One is addressed to the object to be imaged and then to a bucket detector, namely, a detector without spatial resolution, while the other is addressed directly to a spatially resolving detector, without interacting with the object. Neither of the two beams separately contains information on the object absorption profile, nevertheless it can be retrieved exploiting the correlation between them. Since based on the evaluation of second-order momenta of the joint distribution, GI is not a single-shot technique, instead it requires the acquisition of several frames and the signal-to-noise ratio (SNR) scales with the square root of the number of frames [11,12].

Initially motivated by a fundamental debate on the boundary between classical and quantum resources, the attention toward GI is justified by the fact that it can be useful in various practical situations, as in the cases where environmental constraints do not allow placing a fine optical system and pixeled detector behind the object [13], and because it is robust in the presence of atmospheric turbulence or diffusive media on the object path [14-16].

Quantum photon number correlations, a tool of the utmost relevance in quantum technologies [3,11,12,17-23], can add further advantages to GI such as the possibility to probe the sample with a wavelength which differs from the one detected with spatial resolution $[24,25]$, in the case in which the first one is in a range where the imaging system and spatial detection are technologically demanding. Moreover, quantum correlation allows retrieving a ghost image at faint photon flux with a better SNR than by classical beams [25-27], where the stronger than classical correlation can be used also to reject external and detection noise $[18,27,28]$.

Several variations of GI, aiming at increasing its applicability in realistic scenarios, have been proposed, as for example backscattering GI [29], computational GI [30,31], and compressive GI [32,33].

Among them, the so-called "differential GI" (DGI) proposed in 2010 by Ferri et al. [34] has received much attention due to its relevant practical impact, addressing the problem of reconstructing small or faint objects in the field of view. In this situation the conventional GI typically fails because it requires an unaffordable number of acquisitions in order to reach a sufficiently high SNR. Proposed and experimentally realized only with bright thermal light [15,34], DGI does not involve changes in the typical GI optical setup, rather a more efficient use of the available data. In particular, also the integrated signal in the reference channel, from the spatially resolving detector, is used in the data elaboration.

In this work we study the performance of a DGI approach when exploiting quantum resources, motivated by their possible advantage in specific situations, for example in the low-brightness regime. We develop a comprehensive theoretical model of the SNR for both classical and quantum sources, which takes into account nonidealities such as channel inefficiencies and electronic noise of the detector. It becomes known that the brightness of the source, namely the number of photons per spatiotemporal mode, has a fundamental role in determining DGI performances, in terms 
of SNR, together with the photon losses. In particular, for low-brightness sources, the improvement provided by DGI is dramatically affected by photon losses, disappearing or becoming worse than GI for a loss probability larger than $50 \%$. These limitations of the DGI protocol cannot be derived from the classical description of Ref. [34], where correlated beams are treated as identical copies of the same classical stochastic process (an approximation that is suitable only in case of an intense thermal beam).

However, we propose an optimized DGI protocol (ODGI) able to partially compensate for experimental imperfections, retrieving an advantage on GI for any value of losses and brightness. The only further requirement for its application is the characterization of channel efficiencies. This protocol can have a positive impact where it is necessary to keep the photon flux low, as in x-ray GI $[35,36]$. The optimization procedure stems from the one proposed and realized in the absorption estimation framework [37,38].

For demonstrating the performances of this method, we perform an experiment using SPDC light in the low-brightness regime. However, rather than basing the GI reconstruction on temporal coincidences among photon pairs, as in the typical approach of almost all GI experiments with quantum light, here we exploit nonclassical intensity correlation certified by the evaluation of a specific nonclassicality parameter known as the noise reduction factor (NRF) [12,39-42].

We validate the model comparing its prediction with the SNR experimentally estimated for the GI, DGI, and the ODGI protocols. Besides that, in view of real applications, a biological object, a $(285 \mu \mathrm{m})^{2}$ wasp wing detail, is reconstructed with a spatial resolution of $5 \mu \mathrm{m}$.

\section{THEORY}

We consider either an equally split multimode thermal beam (th) or a multimode twin beam (tw) generated by SPDC. In both cases the two beams, used as a probe and reference, respectively, present a spatial correlation [12]. In particular, referring to Fig. 1, the spatial selection performed in the reference beam by the pixel in $x_{j}^{(2)}$ automatically identifies a small area where the correlated probe photons are expected to impinge on the object plane, centered in $x_{j}^{(1)}$. This area represents the spatial resolution of any GI scheme. This can be obtained, for example, if the point-to-point far-field correlations of SPDC are imaged at the detection plane for the reference beam, while at the object plane for the probe beam. A similar condition can be obtained by pairs of correlated spatial modes in split pseudothermal beams. We further assume that the pixel is larger than the coherence area, so that the resolution cells identified by two adjacent pixels do not overlap. Given this one-to-one correspondence between the object plane and the reference detection plane, hereinafter we will omit the suffixes 1 and 2 , identifying $x_{j}^{(2)}$ and $x_{j}^{(1)}$.

Each beam of the twin beam follows thermal statistics, therefore there is no difference between the thermal and twinbeam case when the probe and reference beams are considered separately. In particular, the mean number of photons detected by the pixel in $x_{j}$ is

$$
\left\langle\hat{n}_{2}\left(x_{j}\right)\right\rangle^{\mathrm{th}}=\left\langle\hat{n}_{2}\left(x_{j}\right)\right\rangle^{\mathrm{tw}}=n_{2},
$$

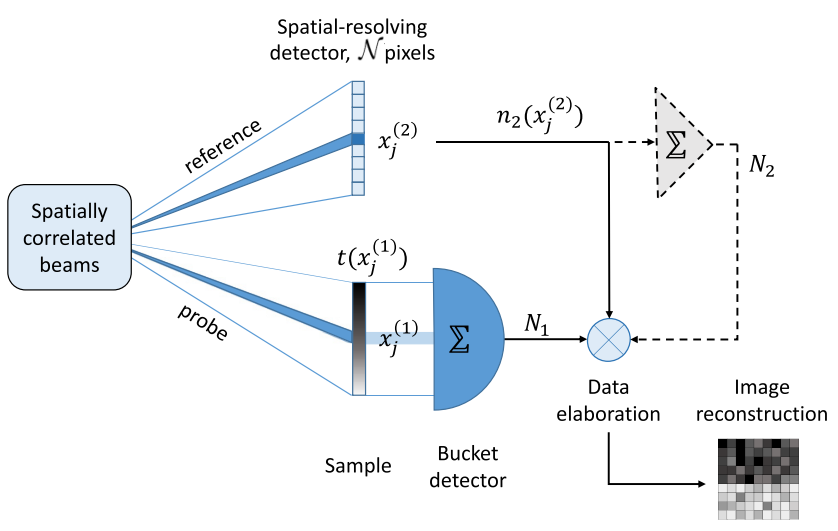

FIG. 1. Scheme of GI and DGI protocols. Spatial correlations between two beams are exploited. The reference beam is detected by a spatial resolving detector, the probe beam, after interacting with the sample impinges on a detector without spatial resolution. Every pixel $x_{j}^{(2)}$ is in one-to-one correspondence with a resolution cell $x_{j}^{(1)}$ at the object plane. For the image reconstruction the number of photons detected by the bucket detector $N_{1}$ and the one detected by each pixel in the resolving detector, $n_{2}\left(x_{j}^{(2)}\right)$, are used in the data elaboration. In DGI protocol (dashed path) also the integrated signal $N_{2}$ is exploited.

where we have assumed a spatially uniform beam. The corresponding number of photons passing through the resolution cell at the object plane and detected by the bucket is

$$
\left\langle\hat{n}_{1}\left(x_{j}\right)\right\rangle^{\text {th }}=\left\langle\hat{n}_{1}\left(x_{j}\right)\right\rangle^{\mathrm{tw}}=n_{2} t\left(x_{j}\right)
$$

with $t\left(x_{j}\right)$ the mean transmission profile of the object in the resolution cell. Note that these expressions are obtained assuming the same loss level on the two channels in absence of the object $\eta_{1}=\eta_{2}=\eta$. This does not reduce the generality of our model since possible unbalancement between the channels can be included in the transmission profile of the object. When $M$ spatiotemporal modes are collected per pixel per frame, the associated variances can be written as [12]

$$
\begin{gathered}
\left\langle\delta^{2} \hat{n}_{2}\left(x_{j}\right)\right\rangle^{\text {th }}=\left\langle\delta^{2} \hat{n}_{2}\left(x_{j}\right)\right\rangle^{\mathrm{tw}} \\
=n_{2}\left(1+n_{2} / M\right)+\Delta_{\mathrm{el}}^{2}, \\
\left\langle\delta^{2} \hat{n}_{1}\left(x_{j}\right)\right\rangle^{\mathrm{th}}=\left\langle\delta^{2} \hat{n}_{1}\left(x_{j}\right)\right\rangle^{\mathrm{tw}} \\
=n_{2} t\left(x_{j}\right)\left[1+n_{2} t\left(x_{j}\right) / M\right]+\Delta_{\mathrm{el}}^{2} .
\end{gathered}
$$

In Eqs. (3) and (4) we have taken into account the electronic noise of the detector, $\Delta_{\mathrm{el}}$. The crucial difference between the thermal and the twin-beam case is in the covariance between two spatially correlated modes [12]:

$$
\begin{gathered}
\left\langle\delta \hat{n}_{2}\left(x_{j}\right) \delta \hat{n}_{1}\left(x_{i}\right)\right\rangle^{\mathrm{th}}=\frac{t\left(x_{i}\right) n_{2}^{2}}{M} \delta_{i, j}, \\
\left\langle\delta \hat{n}_{2}\left(x_{j}\right) \delta \hat{n}_{1}\left(x_{i}\right)\right\rangle^{\mathrm{tw}}=t\left(x_{i}\right)\left(\frac{n_{2}^{2}}{M}+\eta n_{2}\right) \delta_{i, j}
\end{gathered}
$$

It is immediately clear that the correlation is always higher for SPDC light. The ratio of the two expressions is

$$
\frac{\left\langle\delta \hat{n}_{2}\left(x_{j}\right) \delta \hat{n}_{1}\left(x_{j}\right)\right\rangle^{\mathrm{tw}}}{\left\langle\delta \hat{n}_{2}\left(x_{j}\right) \delta \hat{n}_{1}\left(x_{j}\right)\right\rangle^{\mathrm{th}}}=1+\eta \frac{M}{n_{2}}
$$


showing that the correlations, at the base of GI protocols, are significantly stronger for the twin beam for a small number of photons emitted per mode, $n_{2} \ll \eta M$. It follows that, in this regime, only twin-beam light allows object reconstruction in practical situations. In the opposite regime the performance of an equally split thermal light and twin beam is asymptotically the same.

In the conventional GI protocol the transmission profile of the object is retrieved considering the covariance between each pixel of the reference channel $\hat{n}_{2}\left(x_{j}\right)$ and the bucket detector on the other channel, $\hat{N}_{1}=\sum_{i=1}^{\mathcal{N}} \hat{n}_{1}\left(x_{i}\right)$, as represented in Fig. 1. Here, $\mathcal{N}$ is the number of resolution cells at the object plane which corresponds to the number of pixels at the resolving detector. Indeed, from either Eq. (5) or Eq. (6) it becomes known that for both the thermal and the twin-beam case, the reconstructed image is

$$
S_{\mathrm{GI}}\left(x_{j}\right)=\left\langle\delta \hat{N}_{1} \delta \hat{n}_{2}\left(x_{j}\right)\right\rangle \propto t\left(x_{j}\right) .
$$

In [34], Ferri et al. propose the DGI protocol, where the bucket detector signal $\hat{N}_{1}$ is replaced by $\hat{N}_{\text {DGI }}=\hat{N}_{1}-\frac{\left\langle\hat{N}_{1}\right\rangle}{\left\langle\hat{N}_{2}\right\rangle} \hat{N}_{2}$, with $\hat{N}_{2}=\sum_{i=1}^{\mathcal{N}} \hat{n}_{2}\left(x_{i}\right)$ the integrated signal from the spatial resolving detector. This alternative protocol is depicted by the dashed path in Fig. 1 . Note that $\left\langle\hat{N}_{1}\right\rangle /\left\langle\hat{N}_{2}\right\rangle=\bar{t}$, where $\bar{t}$ is the average transmission of the object, i.e., $\bar{t}=(1 / \mathcal{N}) \sum_{i=1}^{\mathcal{N}} t\left(x_{i}\right)$. For DGI the reconstructed image is given by

$$
S_{\mathrm{DGI}}\left(x_{j}\right)=\left\langle\delta \hat{N}_{\mathrm{DGI}} \delta \hat{n}_{2}\left(x_{j}\right)\right\rangle=S_{\mathrm{GI}}\left(x_{j}\right)-\bar{t}\left\langle\delta^{2} \hat{n}_{2}\left(x_{j}\right)\right\rangle .
$$

In the last equality we have used the fact that modes collected by different pixels of the resolving detector are uncorrelated. Making use of Eqs. (3), (5), and (6) we get

$$
\begin{gathered}
S_{\mathrm{DGI}}^{\mathrm{th}}\left(x_{j}\right)=n_{2}\left[\frac{n_{2}}{M} \delta t\left(x_{j}\right)-\bar{t}\right], \\
S_{\mathrm{DGI}}^{\mathrm{tw}}\left(x_{j}\right)=n_{2}\left[\left(\frac{n_{2}}{M}+\eta\right) \delta t\left(x_{j}\right)-\bar{t}(1-\eta)\right] .
\end{gathered}
$$

From Eq. (10), in the limit $n_{2} / M \gg 1$, one retrieves the main feature of DGI as proposed in [34], namely, its sensitivity to the spatial change in the transmission of the object, $\delta t\left(x_{j}\right)=t\left(x_{j}\right)-\bar{t}$, rather than to the absolute value $t\left(x_{j}\right)$. This explains its significant advantages in the reconstruction of small or highly transparent objects. However, it is also clear that in the opposite regime of a small number of photons detected per spatiotemporal mode, the information on the transmission profile of the object is substantially lost. With the twin beam, the transmission profile can be reconstructed even for $n_{2} / M \ll 1$, provided that the efficiency $\eta$ is sufficiently high. Anyway, the possibility to practically get a faithful image is determined by the SNR. It is important to reduce the noise as much as possible on the reconstruction. For this reason, following the method developed in [37,38], we propose a generalization of the DGI protocol by replacing $\hat{N}_{\text {DGI }}$ with $\hat{N}_{k}=\hat{N}_{1}-k \hat{N}_{2}$, leading to

$$
S_{k}\left(x_{j}\right)=\left\langle\delta \hat{N}_{k} \delta \hat{n}_{2}\left(x_{j}\right)\right\rangle=S_{\mathrm{GI}}\left(x_{j}\right)-k\left\langle\delta^{2} \hat{n}_{2}\left(x_{j}\right)\right\rangle,
$$

where $k$ is a constant that can be set in order to minimize the noise in the estimation of $S_{k}\left(x_{j}\right)$, approximately given by

$$
\begin{aligned}
\delta^{2} S_{k}\left(x_{j}\right) & \approx\left\langle\delta^{2} \hat{N}_{k}\right\rangle\left\langle\delta^{2} \hat{n}_{2}\left(x_{j}\right)\right\rangle, \\
\left\langle\delta^{2} \hat{N}_{k}\right\rangle & =\left\langle\delta^{2} \hat{N}_{1}\right\rangle+k^{2}\left\langle\delta^{2} \hat{N}_{2}\right\rangle-2 k\left\langle\delta \hat{N}_{1} \delta \hat{N}_{2}\right\rangle .
\end{aligned}
$$

For $k=0$, i.e., for conventional GI, the variance reduces to $\delta^{2} S_{\mathrm{GI}}\left(x_{j}\right)=\left\langle\delta^{2} \hat{N}_{1}\right\rangle\left\langle\delta^{2} \hat{n}_{2}\left(x_{j}\right)\right\rangle$, and making use of Eqs. (3) and (4),

$$
\delta^{2} S_{\mathrm{GI}}\left(x_{j}\right)=\mathcal{N} n_{2}^{2}\left(\bar{t}+\frac{n_{2}}{M} \bar{t}^{2}\right)\left(1+\frac{n_{2}}{M}\right) .
$$

Equation (14) shows that in the limit of $n_{2} / M \gg 1$ we find the same noise dependence from the mean transmittance squared reported in [34], although the general expression predicts a linear dependence in the opposite regime.

For the sake of simplicity, we focus on the case of a two-level object, with $\epsilon \mathcal{N}$ resolution cells of transmission $t_{-}$ and $(1-\epsilon) \mathcal{N}$ of transmission $t_{+}$. Therefore, $\epsilon$ represents the fraction of the detection area occupied by the lower transmittance portion of the object. Note that $\bar{t}$ can be written in terms of $0 \leqslant \epsilon \leqslant 1$ as $\bar{t}=(1-\epsilon) \mathcal{N} t_{+}+\epsilon \mathcal{N} t_{-}$. Under these assumptions the SNR is defined as

$$
\mathrm{SNR}=\frac{\left|\left\langle S_{+}\right\rangle-\left\langle S_{-}\right\rangle\right|}{\sqrt{\delta^{2}\left\langle S_{+}\right\rangle+\delta^{2}\left\langle S_{-}\right\rangle}},
$$

where $\left\langle S_{ \pm}\right\rangle$and $\delta^{2}\left\langle S_{ \pm}\right\rangle$are, respectively, the mean value of the reconstructed image in correspondence to $t_{ \pm}$and its associated variance. Considering $t_{+}=1, t_{-}=0$, and no electronic noise, the SNRs in the thermal and SPDC case for the GI protocol are:

$$
\begin{aligned}
\mathrm{SNR}_{\mathrm{GI}}^{\mathrm{th}} & =\sqrt{H} \frac{1}{\sqrt{2 \mathcal{N}(1-\epsilon)}} \frac{n_{2}}{n_{2}+M}, \\
\mathrm{SNR}_{\mathrm{GI}}^{\mathrm{tw}} & =\sqrt{H} \frac{1}{\sqrt{2 \mathcal{N}(1-\epsilon)}} \frac{n_{2}+M \eta}{n_{2}+M} .
\end{aligned}
$$

Note that in this case $\epsilon$ simply becomes the fraction of the detection area occupied by the object. In the SNR expression the factor $\sqrt{H}$, being $H$ the number of frames used to estimate $S$, simply comes from the central limit theorem, where the uncertainty on a mean value scales as $\sqrt{H}$, e.g., $\delta^{2}\left\langle S\left(x_{j}\right)\right\rangle=$ $\delta^{2} S\left(x_{j}\right) / H$. From Eqs. (16) and (17) one of the weaknesses of the conventional GI emerges, namely, that for small $\epsilon$ the SNR drops down, making the reconstruction of small objects in the field of view difficult.

In Fig. 2 we report an example of GI reconstruction for a two-level object, for different values of $\epsilon$. From these reconstructions it is possible to experimentally estimate the SNR. $\left\langle S_{ \pm}\right\rangle$and $\delta^{2}\left\langle S_{ \pm}\right\rangle$are estimated as the mean value and the variance on the reconstructed images, in correspondence to the regions of transmittance $t_{ \pm}$, respectively. It can be appreciated how the object, a totally absorbing deposition on the right side of the field of view, better emerges from the noise as $\epsilon$ increases.

This issue of the conventional GI can be overcome by considering the generalized protocol $S_{k}\left(x_{j}\right)$. Indeed, from Eq. (13) we argue that exploiting the spatial correlations between the two beams, i.e., $\left\langle\delta \hat{N}_{1} \delta \hat{N}_{2}\right\rangle \neq 0$, and choosing $k$ appropriately, the noise can be reduced. This idea is at the basis of the optimization procedure we implemented. In particular, we maximize the SNR with respect to $k$, and define the ODGI protocol as

$$
\begin{array}{r}
S_{\mathrm{ODGI}}\left(x_{j}\right)=S_{k_{\mathrm{opt}}}\left(x_{j}\right)=\left\langle\delta \hat{N}_{\mathrm{opt}} \delta \hat{n}_{2}\left(x_{j}\right)\right\rangle, \\
\hat{N}_{\mathrm{opt}}=\hat{N}_{1}-k_{\mathrm{opt}} \hat{N}_{2} .
\end{array}
$$




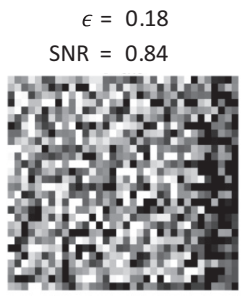

$\epsilon=0.53$

$\mathrm{SNR}=1.13$

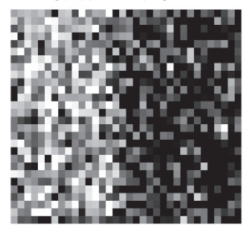

FIG. 2. Experimental GI reconstructions of a binary object ( $t_{+}=1, t_{-}=0$ ) for $\mathcal{N}=952$ and $H=2000$, using SPDC light. The object consists in a totally absorbing deposition occupying a variable fraction $\epsilon$ of the reconstructed area. Different values of $\epsilon$ are considered and the corresponding SNR estimated; for small $\epsilon$, e.g., $\epsilon=0.18$, SNR $<1$ and the deposition on the right side is almost hidden in the noise.

The general expression for $k_{\mathrm{opt}}$, in the twin-beam case, is

$$
k_{\mathrm{opt}}^{\mathrm{tw}}=\frac{n_{2}\left(n_{2}+M \eta\right) \bar{t}}{n_{2}^{2}+M\left(n_{2}+\Delta_{\mathrm{el}}^{2}\right)}
$$

while for the thermal case a similar expression holds but without the term $M \eta$ at the numerator. Focusing on the twinbeam case, we have

$$
\begin{gathered}
n_{2} / M \gg 1: k_{\mathrm{opt}}^{\mathrm{tw}}=\bar{t}=\frac{\left\langle\hat{N}_{1}\right\rangle}{\left\langle\hat{N}_{2}\right\rangle}, \\
n_{2} / M \ll \eta: k_{\mathrm{opt}}^{\mathrm{tw}}=\frac{n_{2}}{n_{2}+\Delta_{\mathrm{el}}^{2}} \eta \frac{\left\langle\hat{N}_{1}\right\rangle}{\left\langle\hat{N}_{2}\right\rangle} .
\end{gathered}
$$

Equation (20) shows that for a high number of detected photons per mode $S_{\text {ODGI }}$ coincides with $S_{\text {DGI }}$. Remarkably, in this case $k_{\text {opt }}$ does not depend on experimental imperfections.

For low-brightness sources, as shown in Eq. (21), $S_{\mathrm{DGI}}$ coincides with $S_{\mathrm{ODGI}}^{\mathrm{tw}}$ only in the ideal case of $\eta=1$ and $\Delta_{\mathrm{el}}=0$; in all other cases ODGI performs better. However, in order to evaluate $k_{\mathrm{opt}}^{\mathrm{tw}}$, absolute values of the channel efficiency $\eta$ and the detection noise $\Delta_{\mathrm{el}}$ should be known $[38,43]$.

The improvement offered by DGI versus the conventional GI protocol can be quantified in terms of SNR. For the sake of simplicity we report the expressions neglecting the electronic noise, namely, $\Delta_{\text {el }}^{2} \ll n_{2}$, and considering a binary object $\left(t_{+}=1, t_{-}=0\right)$ :

$$
\begin{gathered}
n_{2} / M \gg 1 / \epsilon: \frac{\mathrm{SNR}_{\mathrm{DGI}}^{\mathrm{tw}}}{\mathrm{SNR}_{\mathrm{GI}}^{\mathrm{tw}}}=\frac{\mathrm{SNR}_{\mathrm{DGI}}^{\mathrm{th}}}{\mathrm{SNR}_{\mathrm{GI}}^{\mathrm{th}}}=\frac{1}{\sqrt{\epsilon}}, \\
n_{2} / M \ll 1: \frac{\mathrm{SNR}_{\mathrm{DGI}}^{\mathrm{tw}}}{\mathrm{SNR}_{\mathrm{GI}}^{\mathrm{tw}}}=\frac{1}{\sqrt{2\left(\eta-\frac{1}{2}\right)(\epsilon-1)+1}} .
\end{gathered}
$$

These results can be graphically appreciated in Fig. 3. In the high-intensity regime, corresponding to Eq. (22) and reported in Fig. 3(a), SPDC and thermal light show the same perfor-

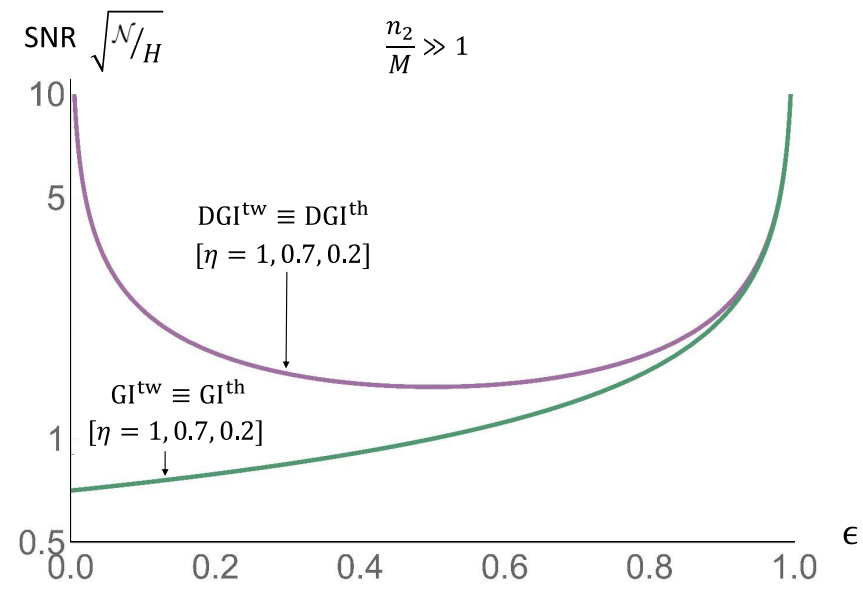

(a)

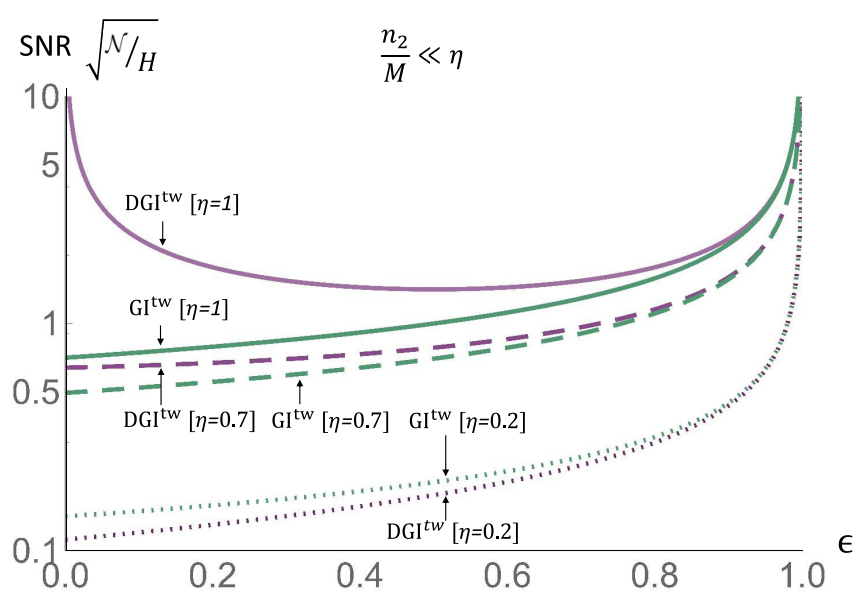

(b)

FIG. 3. SNR, normalized per the number of frames $H$ and the number of pixels in the reconstructed area $\mathcal{N}$, as a function of the object occupation fraction $\epsilon$, for different values of the channel efficiency $\eta\left(t_{+}=1, t_{-}=0, \Delta_{\mathrm{el}}=0\right)$. (a) High-brightness regime. (b) Low- brightness regime, twin-beam case.

mance and the SNRs for both GI and DGI are independent from the channel efficiency. Note that the validity condition of Eq. (22) requires higher and higher values of detected photons per mode as $\epsilon$ decreases. For small $\epsilon$ the DGI advantage is relevant, an extremely interesting feature in view of real applications.

On the contrary, losses in the low-intensity regime have a significant impact. From Eq. (23) it becomes known that, despite that we find the same result of Eq. (22) for $\eta=1$, the improvements offered by DGI over GI decrease with losses. In particular, for $\eta<1 / 2$, DGI performs even worse than the conventional protocol. The dependence of the SNR from the channel efficiency, at low brightness, is evident in Fig. 3(b).

The ODGI protocol can partially compensate for losses; in particular,

$$
n_{2} / M \ll \eta: \frac{\mathrm{SNR}_{\mathrm{ODGI}}^{\mathrm{tw}}}{\mathrm{SNR}_{\mathrm{GI}}^{\mathrm{tw}}}=\frac{1}{\sqrt{\eta^{2}(\epsilon-1)+1}} .
$$




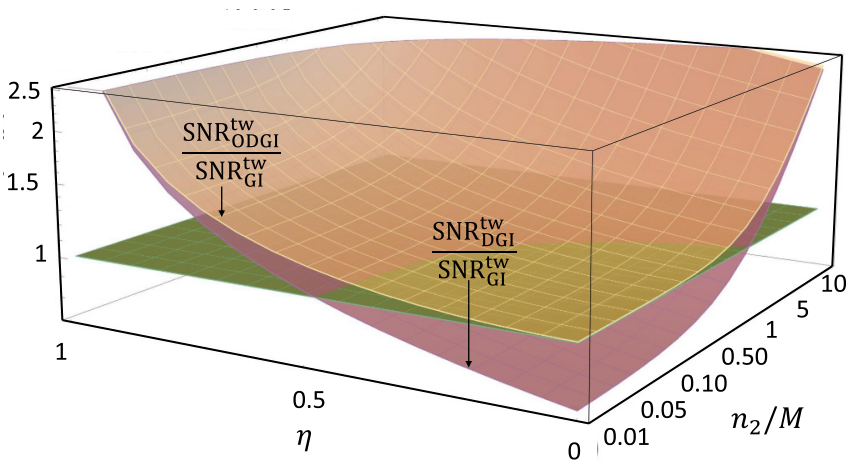

FIG. 4. DGI and ODGI are compared in terms of SNR with GI, varying both the channel efficiency $\eta$ and the number of detected photons per mode $n_{2} / M$. The surfaces are obtained for the twin-beam case, considering a binary object $\left(t_{+}=1, t_{-}=0\right)$ and $\epsilon=0.1$.

From Eq. (24) it emerges that, for low-brightness sources, ODGI always performs better than the conventional GI, but it is also permanently better than DGI, as emerges when comparing Eq. (24) with Eq. (23). Note that in these equations we do not report the expressions for thermal light since in this regime thermal correlations are an order of magnitude weaker than for twin-beam light [see Eq. (7)], thus requiring an unfeasible number of frames to achieve a sufficiently high SNR.

The general performance of DGI and ODGI with respect to conventional GI, for the SPDC case, is reported in Fig. 4 as a function of the channel efficiency $\eta$ and the number of detected photons per mode $n_{2} / M$, fixing $\epsilon=0.1$. Considering the front plane, corresponding to $n_{2} / M=0.01$, one can observe that the highest ODGI advantage over both the other protocols occurs when $\mathrm{SNR}_{\mathrm{DGI}}^{\mathrm{tw}}=\mathrm{SNR}_{\mathrm{GI}}^{\mathrm{tw}}$, i.e., for $\eta \sim 0.5$. Moreover, it emerges that increasing the brightness of the source allows one to obtain an advantage with respect to GI for a lower value of the channel efficiency $\eta$, so that, depending on the experimental condition, the optimal trade-off can be designed.

\section{EXPERIMENT}

Here we present an experiment comparing GI, DGI, and ODGI protocols in the low-brightness regime using a twin beam generated by SPDC.

The experimental setup is reported in Fig. 5. A twinbeam state with degeneracy wavelength $\lambda_{1}=\lambda_{2}=810 \mathrm{~nm}$ is produced pumping a $1-\mathrm{cm}$ type-II beta barium borate (BBO) nonlinear crystal with a continuous-wave laser beam $\left(100 \mathrm{~mW}\right.$ at $\left.\lambda_{p}=405 \mathrm{~nm}\right)$. In this process, with a certain small probability, a photon of the pump is down-converted into two photons. The momentum conservation implies that the two down-converted photons emerge with opposite transverse momenta $\mathbf{q}_{1}=-\mathbf{q}_{2}$. In the far field of the emission, realized at the focal plane of a lens with $f_{F F}=1 \mathrm{~cm}$, momentum correlation is mapped in position correlation, $\mathbf{x}_{1}=-\mathbf{x}_{2}$, with $\mathbf{x}_{i}=\frac{\lambda_{i} \mathbf{q}_{i} f_{F F}}{2 \pi}$. To detect only photons around the degeneracy and to cut the pump, an interference filter with $20 \mathrm{~nm}$ bandwidth centered at $800 \mathrm{~nm}$ is placed after the crystal. To take advantage of the point-to-point far-field correlations, the

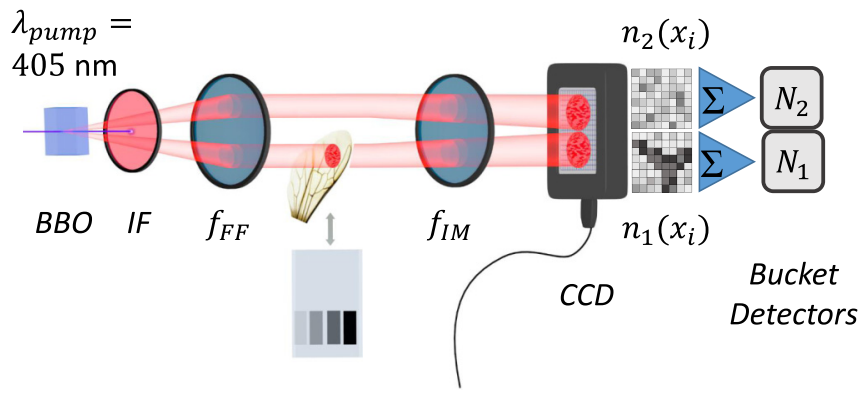

FIG. 5. Scheme of the experimental setup. In the BBO crystal two beams with perfect correlation in the photon number (twin-beam state) are generated. The probe beam interacts with the sample and is detected by a half of a CCD camera chip, while the other beam goes directly to the second half of the chip. The equivalent bucket detector on both channels is simulated by integrating (summing pixels signal) over the two regions. Two different samples are used: A set of uniform depositions of different transmittance $t_{-}$and a biological object, i.e,. a wasp wing.

object is placed directly at the focal plane of the $f_{F F}$ lens, and a second lens with focal length $f_{I M}=1.6 \mathrm{~cm}$, is used to image this plane to the camera sensor, with a magnification factor $M=7.8$.

The detector is a charge-coupled-device (CCD) camera, Princeton Instruments Pixis 400BR Excelon operating in linear mode, i.e., the signal is proportional to the incident number of photons (intensity) in the acquisition time, and cooled down to $-70^{\circ} \mathrm{C}$. It presents high quantum efficiency, nominally $>95 \%$ at $810 \mathrm{~nm}, 100 \%$ fill factor, and low noise [read noise has been estimated to be $\Delta_{\mathrm{el}}=5 e^{-} /(\mathrm{pixel} \cdot$ frame $)$ at $100 \mathrm{kHz}$ acquisition rate, and $\Delta_{\mathrm{el}}=13 e^{-} /($pixel $\cdot$ frame $)$ when the higher $2 \mathrm{MHz}$ digitization rate is used]. The detector area measures $(13.3 \mathrm{~mm})^{2}$ and the size of a physical pixel is $13 \mu \mathrm{m}$; nevertheless, for this experiment we use $3 \times 3$ hardware binning. It is known that the ultimate resolution achievable in ghost imaging is given by the second-order coherence area of the light at the object plane. In particular, following the procedure in [44], we estimated this coherence area to be $(5 \mu \mathrm{m})^{2}$. The binned pixel size corresponds roughly to the coherence area, taking into account the 7.8 magnification factor from the object plane to the detection plane, so that pixels collect independent optical modes thus remaining statistically uncorrelated to each other. Note that in our experimental configuration there is no physical bucket detector. The equivalent bucket detectors on both the beams are simulated by integrating over the respective regions of the sensor.

The average number of photons detected per pixel per frame is $n_{2} \sim 10^{3}$ and the corresponding number of modes is $M=M_{\mathrm{sp}} M_{\mathrm{temp}}$, where $M_{\mathrm{sp}}$ and $M_{\mathrm{temp}}$ are the number of spatial and temporal modes. Being the acquisition time of a single frame is $50 \mathrm{~ms}$, and the coherence time of the SPDC process is around $10^{-12} \mathrm{~s}$, it follows that $M_{\text {temp }} \sim 5 \times 10^{10}$. The dimension of a binned pixel is chosen in order to have $M_{\text {sp }} \sim 1$ incident. It follows that the condition of very low brightness is fulfilled: $n_{2} / M \sim 2 \times 10^{-8} \ll \eta$.

In order to demonstrate the nonclassicality of the detected intensity correlation, we evaluate the NRF parameter defined 
as NRF $=\left\langle\Delta^{2}\left(\hat{n}_{1}-\hat{n}_{2}\right)\right\rangle /\left\langle\hat{n}_{1}+\hat{n}_{2}\right\rangle$, which is the variance of the photon number difference between a pair of correlated pixels normalized by their sum (the shot-noise bound). Only nonclassical correlation allows one to have $0<\mathrm{NRF}<1$ $[3,12]$. In our case we estimated NRF $=0.77 \pm 0.02$. Note that NRF, in our setup, depends on the pixel size with respect to the coherence area, thus it is possible to reach lower values of NRF integrating the signals over larger areas. However, this is not in the scope of the present work.

To experimentally validate the theoretical model we image four different objects, each of them presenting two levels of transmittance $\left(t_{+} \sim 1\right.$ for all cases, $t_{-}=0,0.25,0.34,0.52$, respectively). These samples consist of an antireflectivecoated glass slide with thin metallic depositions. For each sample $3 \times 10^{4}$ frames are acquired at a $100 \mathrm{kHz}$ acquisition rate. In the data processing, a cropped region of $\mathcal{N}=$ $28 \times 34$ binned pixels is reconstructed, including each time of an increasing fraction $\epsilon$ of the low transmittance part of

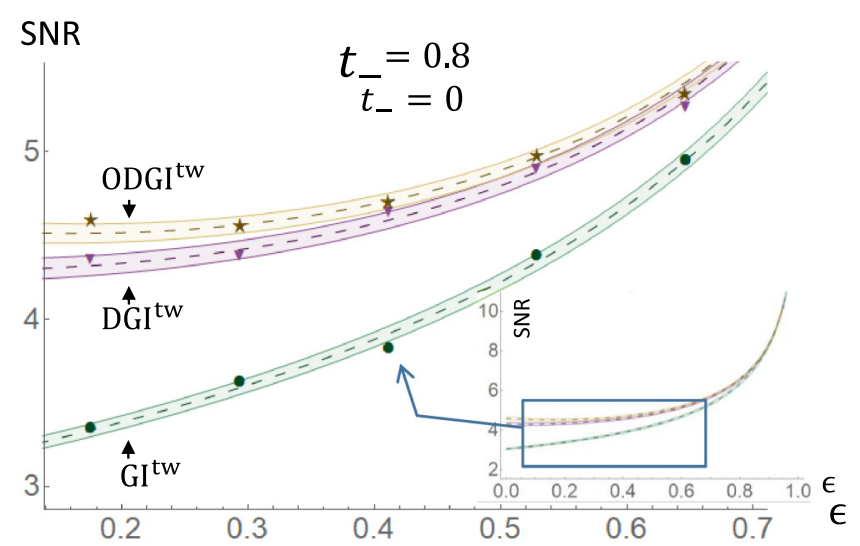

(a)

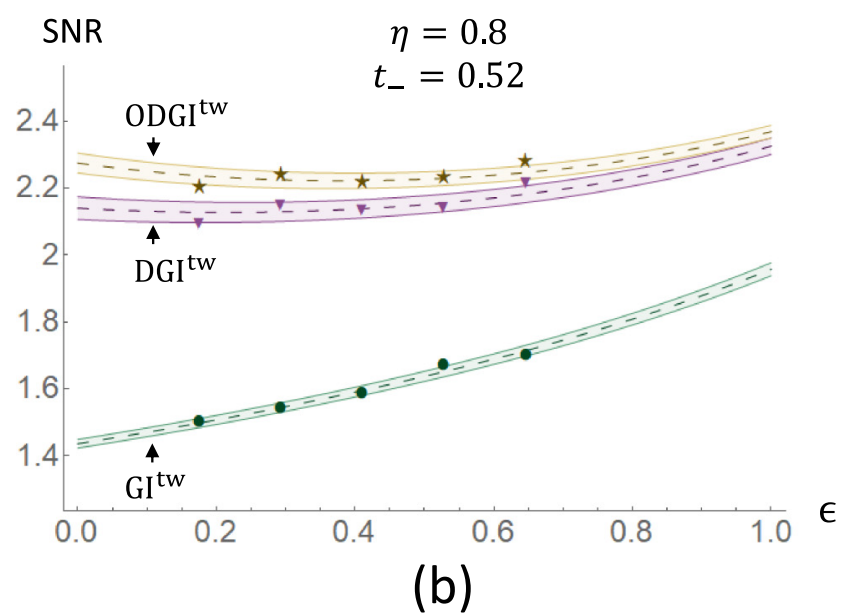

FIG. 6. SNR as a function of $\epsilon$, the fraction of the detection area occupied by the deposition, for (a) $t_{-}=0$ and (b) $t_{-}=0.52$. Green refers to GI, purple to DGI, and yellow to ODGI. The dots are the experimental data, obtained for $H \sim 3 \times 10^{4}$ frames and an area of $\mathcal{N}=952$ pixels. The dashed lines are obtained fitting the data with the theoretical model, considering $\eta$ as a free parameter. The confidence region at $1 \sigma$ is also reported as colored bands around the curves. the object. The reconstruction is performed using the three different protocols $S_{\mathrm{GI}}, S_{\mathrm{DGI}}$, and $S_{\mathrm{ODGI}}$. An example of the corresponding reconstructions is presented in Fig. 2 in the case of the conventional GI. The dependence of SNR, in Eq. (15), from $\epsilon$ is evaluated performing spatial statistics on this kind of image.

Figures 6(a) and 6(b) report the results for two different transmittances, $t_{-}=0$ and $t_{-}=0.52$, respectively. The dashed lines are obtained fitting the experimental data with the theoretical model, considering $\eta$ as a free parameter.

In Fig. 7, at fixed $\epsilon=0.52$, the SNR for different $t_{-}$is reported. Also, in this case data are fitted using the theoretical curves with the efficiency $\eta$ as a free parameter.

For comparing the three protocols in the presence of higher losses, a neutral filter is placed on the twin-beam path. Two different values of channel efficiency are considered: $\eta \sim 0.5$ and $\eta \sim 0.3$. The results corresponding to these experimental situations are reported in Figs. 8 and 9, respectively. In particular, for $\eta=0.3$, the DGI protocol performs worse than the conventional one, while the ODGI always offers an advantage.

In all the cases considered (see Figs. 6-9), the curves fit the experimental data properly, with almost all the data falling in the $1 \sigma$ confidence region. A further element of consistency of the model is the accordance between the value of $\eta$ obtained from the fit and the one independently estimated with the absolute technique described in [43-46], which extends the Klyshko method [47,48]. Indeed, referring, for example, to Fig. 6(a), the values of $\eta$ obtained from the fit and their standard uncertainty are $\eta_{\mathrm{GI}}=0.798 \pm 0.004, \eta_{\mathrm{DGI}}=0.786 \pm$ $0.003, \eta_{\text {ODGI }}=0.786 \pm 0.003$, being the value independently estimated as $\eta=0.794 \pm 0.003$. The values are compatible with a confidence level of $95 \%$. Analogous results are obtained in all the other fits.

Finally, in order to demonstrate that our system can be interesting in view of real application, two different biological

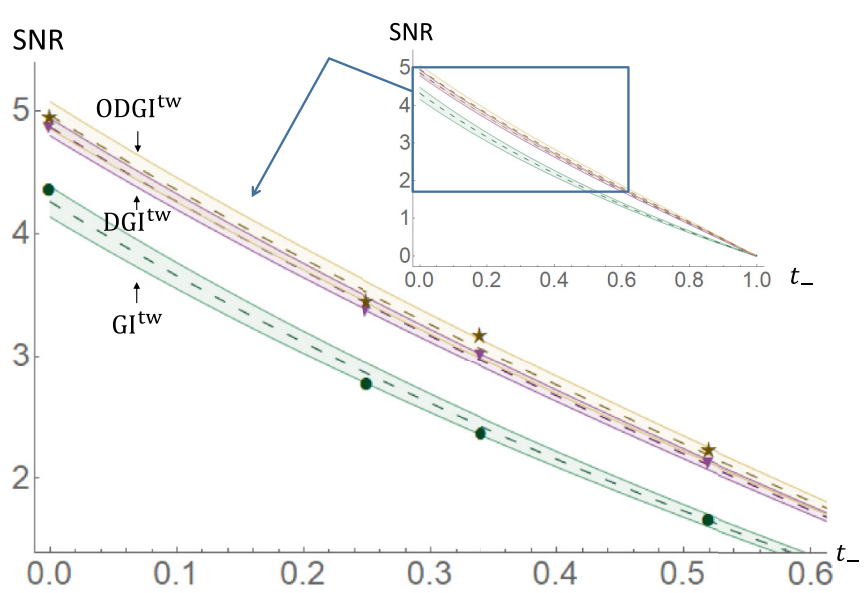

FIG. 7. SNR as a function of $t_{-}$, fixed $\epsilon=0.52$. Green refers to GI, purple to DGI, and yellow to ODGI. The dots are the experimental data, obtained for $H \sim 3 \times 10^{4}$ frames and an area of $\mathcal{N}=952$ pixels. The dashed lines are obtained fitting the data with the theoretical model, considering $\eta$ as a free parameter. The confidence region at $1 \sigma$ is also reported as colored bands around the curves. 


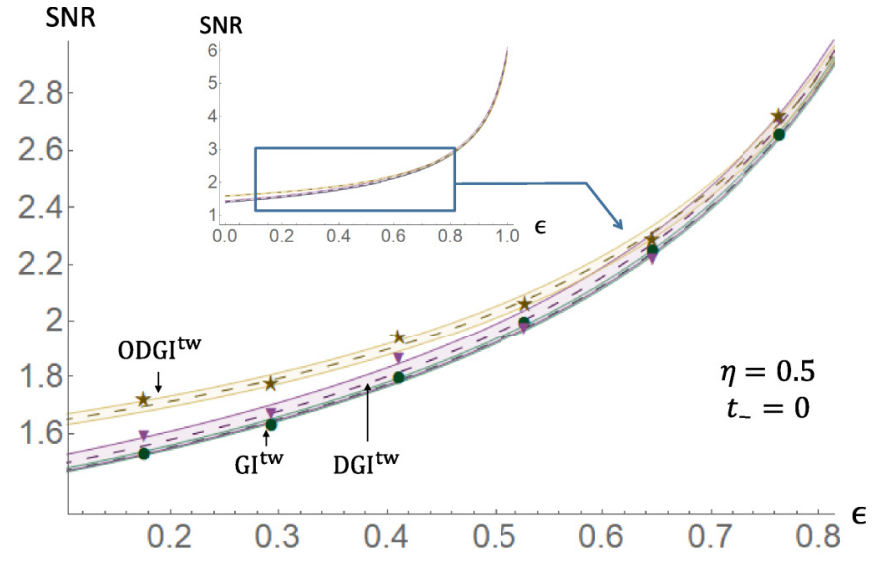

FIG. 8. SNR as a function of $\epsilon$, for $\eta \sim 0.5$. Green refers to GI, purple to DGI, and yellow to ODGI. The dots are the experimental data, obtained acquiring $H \sim 3 \times 10^{4}$ frames and considering an area of $\mathcal{N}=952$ pixels. The dashed lines are obtained fitting the data with the theoretical model, considering $\eta$ as a free parameter. The confidence region at $1 \sigma$ is also reported as colored bands around the curves.

samples are imaged, in particular, a Polistes wasp wing and a green bug wing. The details cover a region of $\mathcal{N}=57 \times 57$ binned pixels, corresponding to an area of $(285 \mu \mathrm{m})^{2}$ in the object plane. The resolution achieved with our setup is $5 \mu \mathrm{m}$, corresponding to the size of the second-order coherence area at the object plane reached in the actual setup. The resolution is not the main concern of our paper, focused on the SNR, but we mention that it is aligned with another recent ghost microscopy experiment [25]. In Fig. 10 the Polistes wasp wing reconstructions, obtained for $2 \times 10^{5}$ frames acquired at $2 \mathrm{MHz}$, are reported. Figure 10(a) is the direct image. To obtain the reconstructed images via $S_{\mathrm{GI}}$ and $S_{\mathrm{ODGI}}$, in Figs. 10(b) and 10(c), respectively, the total region is divided

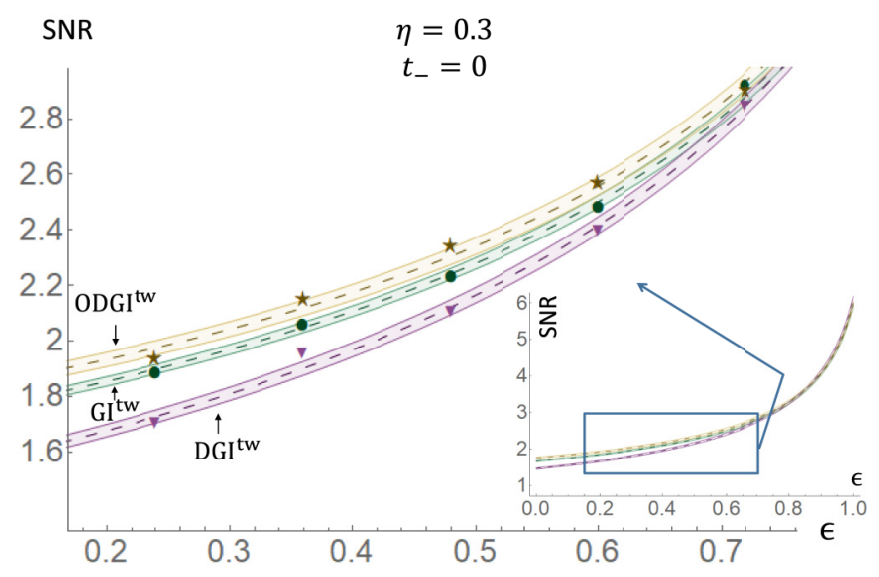

FIG. 9. SNR as a function of $\epsilon$, for $\eta \sim 0.3$. Green refers to GI, purple to DGI, and yellow to ODGI. The dots are the experimental data, obtained acquiring $H \sim 3.5 \times 10^{4}$ frames and considering an area of $\mathcal{N}=525$ pixels. The dashed lines are obtained fitting the data with the theoretical model, considering $\eta$ as a free parameter. The confidence region at $1 \sigma$ is also reported as colored bands around the curves.

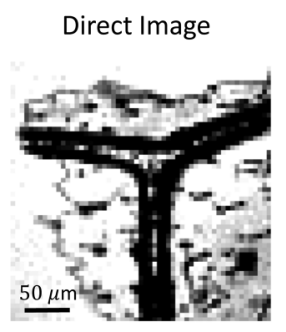

(a)

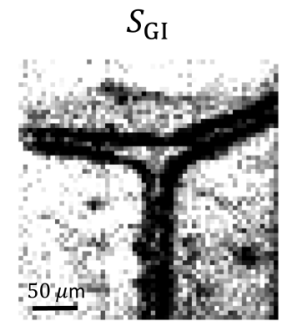

(b)

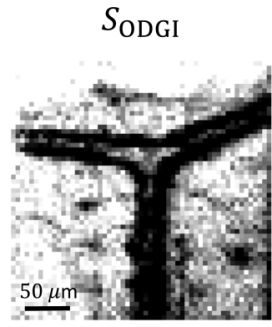

(c)
FIG. 10. Images of a wasp wing with spatial resolution of $(5 \mu \mathrm{m})^{2}$. (a) Direct image, obtained averaging 5000 frames. (b), (c) Reconstruction using the GI and ODGI protocol, respectively. The total region is divided into nine subregions, the protocol is applied to each of them, and finally the complete image is recovered. Forty blocks of 5000 images acquired at $2 \mathrm{MHz}$ are processed.

into nine subregions and the protocol applied to each of them. At this digitization rate the presence of higher electronic noise lowers the improvement offered by ODGI, by the way a few more details can be appreciated in Fig. 10(c) rather than in Fig. 10(b) (see, e.g., the increased visibility of the edge of the wing in the left side). In Fig. 11(b) the reconstruction of the green bug wing is shown [Fig. 11(a) is the direct image]. In this case we acquired $4 \times 10^{4}$ frames at $100 \mathrm{kHz}$. In particular, in Figs. 11(c) and 11(d) we report the reconstruction of two different details. In Fig. 11(c), the edge of the wing is more defined when using $S_{\text {ODGI }}$ with respect to the conventional (a)

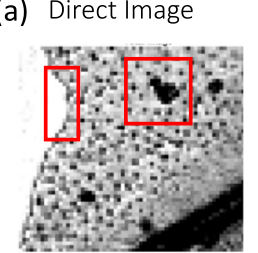

(b) ODGI

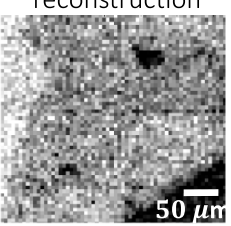

(c)

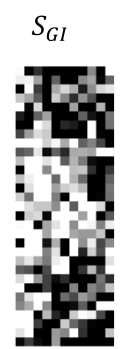
$S_{O D G I}$ (d)
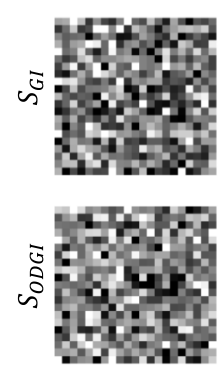

10.000
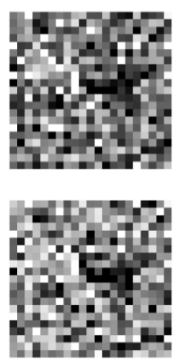

20.000
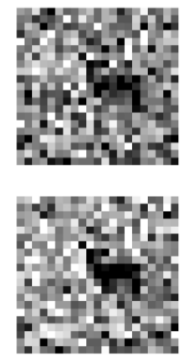

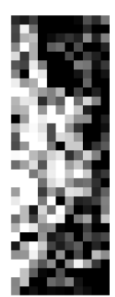

40.000
FIG. 11. Images of a green bug wing with spatial resolution of $(5 \mu \mathrm{m})^{2}$. (a) Direct image, obtained averaging 5000 frames. (b) Reconstruction using the ODGI protocol. The total region is divided into nine subregions, the protocol is applied to each of them, and finally the complete image is recovered. Eight blocks of 5000 images acquired at $100 \mathrm{kHz}$ are processed. (c) $S_{\mathrm{GI}}$ and $S_{\mathrm{ODGI}}$ reconstruction of the border detail squared in (a). (d) $S_{\mathrm{GI}}$ and $S_{\mathrm{ODGI}}$ reconstruction of the spot detail squared in (a). The reconstruction for different number of frames is reported. 
protocol. In Fig. 11(d) the $S_{\mathrm{GI}}$ and $S_{\mathrm{ODGI}}$ reconstructions of a second detail are reported for different numbers of frames: It can be appreciated that using the ODGI protocol the spot emerges from the noise for a lower number of frames. In particular, note that the detail for 40000 frames using $S_{\mathrm{GI}}$ is comparable with the one obtained for 20000 by $S_{\text {ODGI }}$. This result is in agreement with the prediction of our model for these specific conditions. This means that the photon dose can be reduced to a half while providing the same information; an important improvement when considering delicate samples (e.g., for x-ray ghost imaging).

\section{CONCLUSION}

In this work, we extended the differential ghost imaging protocol [34] in the unexplored regime of low-brightness sources and quantum correlation. The attention toward the DGI is justified since it offers significant SNR improvements over the conventional GI, in particular in the presence of small or highly transparent objects. However, this advantage has been demonstrated in previous works within the implicit assumption of classical intense thermal beams. In this paper, in particular we point to the investigation of the lowintensity regime, including quantum resources, which is of great interest since there are practical situations where it is worth keeping the photon dose low, e.g., biological, delicate, or photosensitive samples. For example, this represents an issue in x-ray imaging and spectroscopy [35,36]. We note that in this contest, improving the SNR performance at fixed exposure time, i.e., without increasing the photon dose, is of utmost importance.

A theoretical model in terms of experimental quantities has been developed, both for thermal and SPDC light, for any value of the source brightness. Also experimental imperfections such as losses and electronic noise in the detector have been considered. It becomes known that DGI performances in the regime of a small number of photons per spatiotemporal mode $\left(n_{2} / M \ll \eta\right)$ are highly affected by the experimental imperfections. For example, the DGI advantage in terms of
SNR is almost washed away even for relatively high efficiency such as the one in our setup, $\eta=0.8$, and the model shows that for lower efficiencies, namely, $\eta<0.5$, DGI is worse than GI.

Therefore, inspired by what is done in the absorption estimation framework in [37], we propose an optimized protocol (ODGI) able to partially compensate for the detrimental effects of experimental imperfections such as channel efficiencies and electronic noise, but requiring their estimation. In the high-brightness regime ODGI is always equivalent to the original DGI protocol. In the opposite, low-brightness case it coincides with DGI when $\eta=1$ while in the realistic condition of $\eta<1$ it always performs better than both DGI and GI.

The theoretical model has been experimentally validated in the low brightness regime using quantum correlated beams produced by SPDC. The number of photons collected per pixel was $n_{2} \sim 10^{3}$ : This regime allows one to reconstruct the images by performing intensity correlations, without the need of any time-coincidence scheme. Finally, in view of possible real applications, the optimized protocol has been successfully employed in the reconstruction of a complex biological object, demonstrating that a reduction of the photon dose is possible while maintaining the same SNR of the conventional GI protocol.

\section{ACKNOWLEDGMENTS}

The authors acknowledge the European Union's Horizon 2020 and the EMPIR Participating States in the context of Project 17FUN01 BeCOMe for financial support. The authors also thank Matteo Fretto who realized the absorbing samples used. I.R.-B. and E.L. conceived the idea and developed the theoretical model of this work, which was discussed and designed with input from all the authors. E.L., A.M., A.A., and O.S. realized the experimental setup and collected the data in INRIM quantum optics labs (coordinated by M.G.). All authors discussed the results and contributed to the writing of the paper.

The authors declare no conflicts of interest.
[1] A. V. Belinskii and D. N. Klyshko, Two-photon optics: Diffraction, holography, and transformation of two-dimensional signals, Zh. Eksp. Teor. Fiz. 105, 487 (1994) [Sov. Phys. JETP 78, 259 (1994)].

[2] T. B. Pittman, Y. H. Shih, D. V. Strekalov, and A. V. Sergienko, Optical imaging by means of two-photon quantum entanglement, Phys. Rev. A 52, R3429(R) (1995).

[3] I. Ruo-Berchera and I. P. Degiovanni, Quantum imaging with sub-Poissonian light: Challenges and perspectives in optical metrology, Metrologia 56, 024001 (2019).

[4] A. Gatti, E. Brambilla, M. Bache, and L. A. Lugiato, Ghost Imaging with Thermal Light: Comparing Entanglement and Classical Correlation, Phys. Rev. Lett. 93, 093602 (2004).

[5] F. Ferri, D. Magatti, A. Gatti, M. Bache, E. Brambilla, and L. A. Lugiato, High-Resolution Ghost Image and Ghost Diffraction Experiments with Thermal Light, Phys. Rev. Lett. 94, 183602 (2005).
[6] A. Valencia, G. Scarcelli, M. D’Angelo, and Y. Shih, TwoPhoton Imaging with Thermal Light, Phys. Rev. Lett. 94, 063601 (2005).

[7] X. H. Chen, I. N. Agafonov, K. H. Luo, Q. Liu, R. Xian, M. V. Chekhova, and L. A. Wu, High-visibility, high-order lensless ghost imaging with thermal light, Opt. Lett. 35, 1166 (2010).

[8] Y. H. Zhai, X. H. Chen, D. Zhang, and L. A. Wu, Two-photon interference with true thermal light, Phys. Rev. A 72, 043805 (2005).

[9] S. Hartmann, A. Molitor, and W. Elsasser, Ultrabroadband ghost imaging exploiting optoelectronic amplified spontaneous emission and two-photon detection, Opt. Lett. 40, 5770 (2015).

[10] J. H. Shapiro and R. W. Boyd, Phys. Ghost Imaging, Quantum Inf. Process. 11, 949 (2012).

[11] M. Genovese, Real applications of quantum imaging, J. Opt. 18, 073002 (2016).

[12] A. Meda, E. Losero, N. Samantaray, F. Scafirimuto, S. Pradyumna, A. Avella, I. Ruo-Berchera, and M. Genovese, 
Photon-number correlation for quantum enhanced imaging and sensing, J. Opt. 19, 094002 (2017).

[13] A. Meda, A. Caprile, A. Avella, I. Ruo-Berchera, I. P. Degiovanni, A. Magni, and M. Genovese, Magneto-optical imaging technique for hostile environments: The ghost imaging approach, Appl. Phys. Lett. 106, 262405 (2015).

[14] W. Gong and S. Han, Correlated imaging in scattering media, Opt. Lett. 36, 394 (2011).

[15] M. Bina, D. Magatti, M. Molteni, A. Gatti, L. A. Lugiato, and F. Ferri, Backscattering Differential Ghost Imaging in Turbid Media, Phys. Rev. Lett. 110, 083901 (2013).

[16] R. E. Meyers, K. S. Deacon, A. D. Tunick, and Y. Shih, Virtual ghost imaging through turbulence and obscurants using Bessel beam illumination, Appl. Phys. Lett. 100, 061126 (2012).

[17] D. G. Monticone, K. Katamadze, P. Traina, E. Moreva, J. Forneris, I. Ruo-Berchera, P. Olivero, I. P. Degiovanni, G. Brida, and M. Genovese, Beating the Abbe Diffraction Limit in Confocal Microscopy via Nonclassical Photon Statistics, Phys. Rev. Lett. 113, 143602 (2014).

[18] E. D. Lopaeva, I. Ruo-Berchera, I. P. Degiovanni, S. Olivares, G. Brida, and M. Genovese, Experimental Realization of Quantum Illumination, Phys. Rev. Lett. 110, 153603 (2013).

[19] G. Brida, M. Genovese, and I. Ruo-Berchera, Experimental realization of sub-shot-noise quantum imaging, Nat. Photonics 4, 227 (2010).

[20] S. T. Pradyumna, E. Losero, I. Ruo-Berchera, P. Traina, M. Zucco, C. S. Jacobsen, U. L. Andersen, I. P. Degiovanni, M. Genovese, and T. Gehring, Quantum-enhanced correlated interferometry for fundamental physics tests, arXiv:1810.13386.

[21] E. Knyazev, F. Y. Khalili, and M. V. Chekhova, Overcoming inefficient detection in sub-shot-noise absorption measurement and imaging, Opt. Express 27, 7868 (2019).

[22] Z. Zhang, M. Tengner, T. Zhong, F. N. Wong, and J. H. Shapiro, Entanglement's Benefit Survives an Entanglement-Breaking Channel, Phys. Rev. Lett. 111, 010501 (2013).

[23] J. Perina, Jr., O. Haderka, A. Allevi, and M. Bondani, Absolute calibration of photon-number-resolving detectors with an analog output using twin beams, Appl. Phys. Lett. 104, 041113 (2014).

[24] K. W. Clifford Chan, M. N. O'Sullivan, and R. W. Boyd, Twocolor ghost imaging, Phys. Rev. A 79, 033808 (2009).

[25] R. S. Aspden et al., Photon-sparse microscopy: Visible light imaging using infrared illumination, Optica 2, 1049 (2015).

[26] G. Brida, M. V. Chekhova, G. A. Fornaro, M. Genovese, E. D. Lopaeva, and I. Ruo Berchera, Systematic analysis of signalto-noise ratio in bipartite ghost imaging with classical and quantum light, Phys. Rev. A 83, 063807 (2011).

[27] P. A. Morris, R. S. Aspden, J. E. C. Bell, R. W. Boyd, and M. J. Padgett, Imaging with a small number of photons, Nat. Commun. 6, 5913 (2011).

[28] D. A. Kalashnikov, Z. Pan, A. I. Kuznetsov, and L. A. Krivitsky, Quantum Spectroscopy of Plasmonic Nanostructures, Phys. Rev. X 4, 011049 (2014).

[29] R. Meyers, K. S. Deacon, and Y. Shih, Ghost-imaging experiment by measuring reflected photons, Phys. Rev. A 77, 041801 (2008).

[30] J. H. Shapiro, Computational ghost imaging, Phys. Rev. A 78, 061802(R) (2008).
[31] Y. Bromberg, O. Katz, and Y. Silberberg, Ghost imaging with a single detector, Phys. Rev. A 79, 053840 (2009).

[32] O. Katz, Y. Bromberg, and Y. Silberberg, Compressive ghost imaging, Appl. Phys. Lett. 95, 131110 (2009).

[33] W. Yu, Adaptive compressive ghost imaging based on wavelet trees and sparse representation, Opt. Express 22, 7133 (2014).

[34] F. Ferri, D. Magatti, L. A. Lugiato, and A. Gatti, Differential Ghost Imaging, Phys. Rev. Lett. 104, 253603 (2010).

[35] A. X. Zhang, Y. H. He, L. A. Wu, L. M. Chen, and B. B. Wang, Tabletop X-ray ghost imaging with ultra-low radiation, Optica 5, 374 (2018).

[36] A. Schori and S. Shwartz, X-ray ghost imaging with a laboratory source, Opt. Express 25, 14822 (2017).

[37] P.-A. Moreau, J. Sabines-Chesterking, R. Whittaker, S. K. Joshi, P. M. Birchall, A. McMillan, J. G. Rarity, and J. C. F. Matthews, Demonstrating an absolute quantum advantage in direct absorption measurement, Sci. Rep. 7, 6256 (2017).

[38] E. Losero, I. Ruo-Berchera, A. Meda, A. Avella, and M. Genovese, Unbiased estimation of an optical loss at the ultimate quantum limit with twin-beams, Sci. Rep. 8, 7431 (2018).

[39] G. Brida, L. Caspani, A. Gatti, M. Genovese, A. Meda, and I. Ruo-Berchera, Measurement of Sub-Shot-Noise Spatial Correlations Without Background Subtraction, Phys. Rev. Lett. 102, 213602 (2009).

[40] M. Bondani and A. Allevi, Can nonclassical correlations survive in the presence of asymmetric lossy channels? Eur. Phys. J. D 72, 178 (2018).

[41] J. Peřina, Jr., V. Michálek, and O. Haderka, Reconstruction of Joint Photon-Number Distributions of Twin Beams Incorporating Spatial Noise Reduction, Phys. Rev. Appl. 10, 064054 (2018).

[42] T. S. Iskhakov, V. C. Usenko, R. Filip, M. V. Chekhova, and G. Leuchs, Low-noise macroscopic twin beams, Phys. Rev. A 93, 043849 (2016).

[43] A. Avella, I. Ruo-Berchera, I. P. Degiovanni, G. Brida, and M. Genovese, Absolute calibration of an EMCCD camera by quantum correlation, linking photon counting to the analog regime, Opt. Lett. 41, 1841 (2016).

[44] A. Meda, I. Ruo-Berchera, I. P. Degiovanni, G. Brida, M. L. Rastello, and M. Genovese, Absolute calibration of a chargecoupled device camera with twin beams, Appl. Phys. Lett. 105, 101113 (2014).

[45] G. Brida, M. Genovese, I. Ruo-Berchera, M. Chekhova, and A. Penin, Possibility of absolute calibration of analog detectors by using parametric downconversion: A systematic study, J. Opt. Soc. Am. B 23, 2185 (2006).

[46] G. Brida, I. P. Degiovanni, M. Genovese, M. L. Rastello, and I. Ruo-Berchera, Detection of multimode spatial correlation in PDC and application to the absolute calibration of a CCD camera, Opt. Express 18, 20572 (2010).

[47] G. Brida, S. Castelletto, I. P. Degiovanni, C. Novero, and M. L. Rastello, Quantum efficiency and dead time of single-photon counting photodiodes: A comparison between two measurement techniques, Metrologia 37, 625 (2000).

[48] D. N. Klyshko, Use of two-photon light for absolute calibration of photoelectric detectors, Sov. J. Quantum Electron. 10, 1112 (1980). 